\title{
Amendment of the measurement of the equivalent series resistance at high frequencies
}

Huimin $\mathrm{Liu}^{1,2}$, Baoquan $\mathrm{Ai}^{1}$, and Fengguo $\mathrm{Li}^{1 *}$

${ }^{1}$ National Demonstration Center for Experimental Physics Education, 510006 Guangzhou, China

${ }^{2}$ Huizhou High School, 516005 Huizhou, China

* Corresponding Author: Lifengguo@m.scnu.edu.cn

(Received: 10/10/2019; Accepted: 06/29/2020; Published: 09/29/2020)

DOI: https://doi.org/10.37906/real.2020.6

\begin{abstract}
A new method is proposed in (Ivković, Marković, Ivković, \& Cvetanović, 2017) to measure the equivalent series resistance (ESR) and the inductance of a coil at low frequencies. However, a special phenomenon has appeared at high frequencies. It is found that the intercept has appeared and the measured inductance value had a large deviation from its nominal value. According to the analysis of result, an amended method is proposed in this paper. The ESR should be written as $E S R^{\prime}=E S R+b$. The inductance value should be amended according to the linear relation between the measured value and the nominal value.
\end{abstract}

Keywords: ESR, inductance, intercept, amendment

\section{Introduction}

The capacitors and inductors of electric circuits in reality, when they are directly used without any adjustment, are not ideal components. They can be treated as ideal capacitors and inductors when be in series with a resistance, which is defined as the equivalent series resistance (ESR). The ESR always is an $\mathrm{AC}$ resistance and can be measured at specified frequencies. The recent research (Ivković, Marković, Ivković, \& Cvetanović, 2017) has presented a new method to measure the ESR of the capacitor and inductance in a LCR circuit. When the ratio of capacitance to a given frequency range is a constant, the ESR can be write as a proportional function and it is,

$$
E S R=K T,
$$

where $K$ is a coefficient and $T$ is the period of oscillations. ESR can be measured by examining the underdamped oscillations of the LCR circuit. The decay time $\tau$ of the underdamped oscillations is calculated according to the equation $\tau=2 L / R$, where $R$ is the total ohmic resistance of the circuit and $L$ is the coil inductance. When $\tau$ is measured, ESR will be obtained:

$$
E S R=\frac{2 L}{\tau}-R_{t},
$$

where $R_{t}$ is the ohmic resistance. From Equations (1) and (2), when n capacitors are connected in parallel, the relation can be written as,

$$
\frac{T_{n}}{n}=\frac{2 L}{K} \frac{1}{\tau_{n}}-\frac{R_{t}}{K},
$$


where $n$ means the number of capacitors, $T_{n}$ and $\tau_{n}$ correspond to a given number of $n$, respectively. Noted that $K$ is not used to calculate $L$. By plotting a relationship figure between $T_{n} / n$ and $\tau_{n}$, the slope and the intercept of the Equation (3) can be obtained. As a result, the value of $L$ can be calculated.

It can be clearly seen from the graph of figure 7 in Reference (Ivković, Marković, Ivković, \& Cvetanović, 2017), which the dependence ESR on $T$ is linear when the frequency below $3300 \mathrm{~Hz}$. Correspondingly, the extrapolated linear fit passes through the point $(0,0)$, which indicates that the ESR is directly proportional to $T$. However, for the higher frequencies, namely, for $T$ values lower than $320 \mu$ s, the dependence obviously deviates from the line, so the presumptions of the method no longer stand. Motivated by this special phenomenon, we will repeat the experiments with the method proposed in (Ivković, Marković, Ivković, \& Cvetanović, 2017) at high frequencies and focus on finding an amended method to measure ESR in the Equation (2) and $L$ in the Equation (3).

\section{The intercept of ESR and its amendment}

We measured the $100 \mathrm{nF}$ and 10nF capacitors of Class1, and the $22 \mathrm{nF}$ and $3.3 \mathrm{nF}$ capacitors of Class2, respectively. Inductance coils (GX9/4) varied from $10 \mathrm{mH}$ to $1 \mathrm{H}$, and the corresponding ohmic resistance varied from $5.7 \Omega$ to $184.1 \Omega$. The square-wave voltage of the signal generator (SP1641B) was $13 \mathrm{~V}_{\mathrm{PP}}$, and the frequency was $50 \mathrm{~Hz}$. An analog oscilloscope (GOS-620, $20 \mathrm{MHz}$ bandwidth) was used to measure the voltage on the capacitor. Before the measurement, the samples of capacitors were carefully chosen by a digital multi-meter (DT-9205A). The results are showed in Figure 1.

Figure 1 shows ESR and its fitting line with the change of $T$ in different values of the capacitors. It is found that the ESR and $T$ have a good linear relation. However, the intercept obviously appears, which means that the ESR is not directly proportional to $T$. The intercept is 41 for the $22 \mathrm{nF}$ capacitor of Class2, while the intercept is 50 for the $100 \mathrm{nF}$ capacitor of Class1. Since the intercept is far greater than zero, ESR cannot be calculated by the Equation (2). Therefore, ESR should be amended. If the intercept of the fitting line is $b$, the relation of ESR and $T$ should be written as

$$
E S R=K T+b .
$$
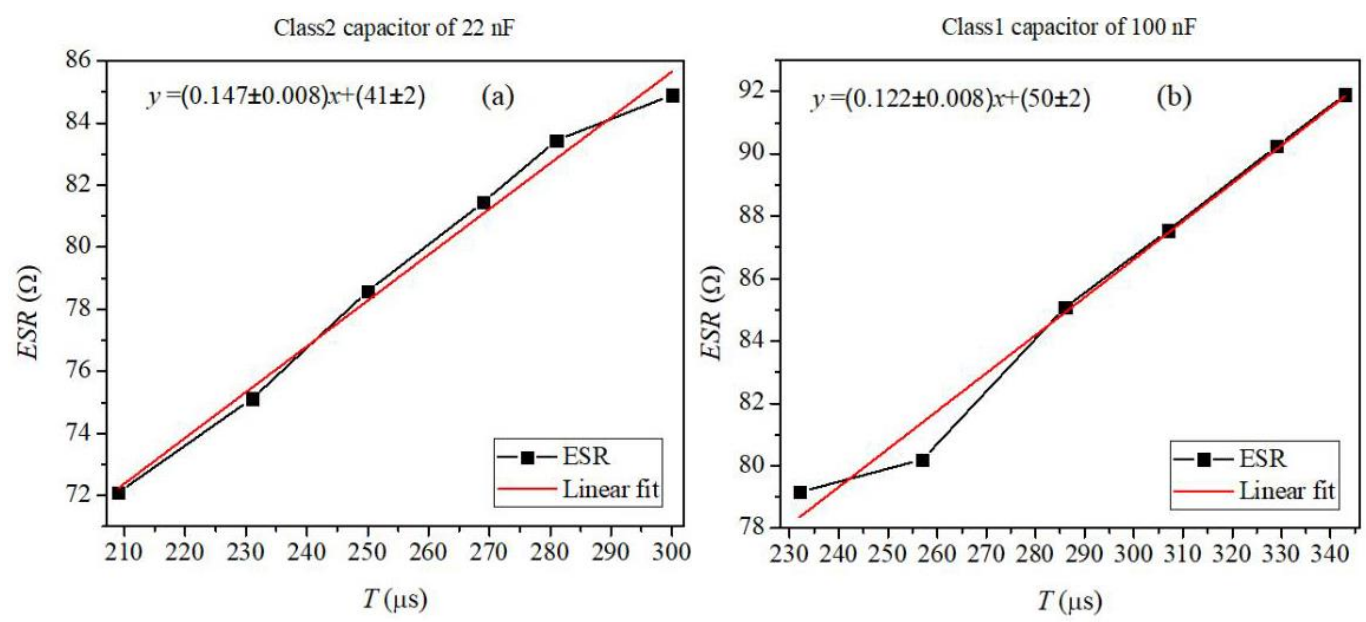

Figure 1. (color online). (a) Graph of $\mathrm{ESR}=f(T)$ of $22 \mathrm{nF}$ and its fitting line. (b) Graph of $\mathrm{ESR}=f(T)$ of $100 \mathrm{nF}$ and its fitting line. 
In order to satisfy the condition that a fitting line should pass through $(0,0)$, we can write $K T$ in the form of $K T=E S R^{\prime} . E S R^{\prime}$ is seen as the amended value of ESR and it is equal to ESR- $b$. Correspondingly, by substituting $E S R^{\prime}$ to the Equation (2), we can also obtain the amended inductance $L^{\prime}$ and the amended resistance $R_{t}^{\prime}$, respectively:

$$
\begin{aligned}
& L^{\prime}=\frac{\left(E S R^{\prime}+R_{t}\right) \tau}{2}, \\
& R_{t}^{\prime}=\frac{2 L}{\tau}-E S R^{\prime} .
\end{aligned}
$$

Note that the values of $R_{t}$ and $L$ in Equation (2) are constant. They can be obtained by direct measurement. Here we will try to explain why the intercept exist. It is well known that in the actual circuit, the equivalent series inductance (ESL) is existing, which models the inductance of the capacitor leads in series with the equivalent inductance of the capacitor plates. These factors can lead to the existence of the intercept and influence measured values of $R_{t}$ and $L$. They are commonly difficult to obtain by measuring. According to results, when the intercept $b>0, R_{t}^{\prime}$ is great than $R_{t}$. In this case, it can be assured that lead resistances contribute more to the intercept. When $b<0, L^{\prime}$ is larger than $L$, ESL may affect the true value of $L$.

We take the first point $(209,72)$ in figure 1 (a) for example. The intercept of the fitting line is $b=41$. The point corresponds to a nominal value of $L=50 \mathrm{mH}$, and ohmic resistance $R_{t}=12 \Omega$. If we substitute $b, L$, and $R_{t}$ into the Equation (2), we can get its $E S R=72 \Omega$. The amended value of $E S R^{\prime}=31 \Omega$ can be obtained from the Equation (4). The amended value is far less than ESR. Meanwhile, the amended value $R_{t}^{\prime}=53 \Omega$ is larger than $R_{t}=12 \Omega$. It indicates that the lead resistances have more contribution to the intercept.

\section{Amendment for the value of inductance}

Figure 2(a) shows the graph of the $T_{n} / n=f\left(1 / \tau_{n}\right)$ functional dependence, obtained in our experiment. By substituting the slope and intercept of $T_{n} / n=f\left(1 / \tau_{n}\right)$ into Equation (3), we can get the measured value of inductance. As can be seen from figure 2(a), the graph shows that $T_{n} / n$ and $1 / \tau_{n}$ have an obvious linear relation, so the measured value of inductance can be obtained by Equation (3), which means that the measured value should be in good agreement with the nominal value. Unfortunately, measured values of the inductance are always larger than nominal values. For example, the nominal value $L_{N}$ of the inductor is $20 \mathrm{mH}$, while the measured value $L_{M}$ is $71 \mathrm{mH}$ when we connect capacitors of Class2 $22 \mathrm{nF}$ in parallel. The measured value is far greater than the nominal value.

To further investigate this deviation, nominal values of inductors from $20 \mathrm{mH}$ to $90 \mathrm{mH}$, as well as from $0.1 \mathrm{H}$ to $0.5 \mathrm{H}$ were measured by capacitors. These values of capacitors were Class $1100 \mathrm{nF}$ and $10 \mathrm{nF}$; Class2 $22 \mathrm{nF}$ and $3.3 \mathrm{nF}$. The results are shown in figures 2(b), 2(c), and 2(d). As can be seen from figures 2(b), 2(c), and 2(d), measured values are also greater than nominal values. Fortunately, nominal values (abscissa) and measured values (ordinate) have a good linear relation. It provides a possible way that the deviation between the measured value and the nominal value can be amended if the relation between nominal values $L_{N}$ and measured values $L_{M}$ is obtained.

In generally, the relation between $L_{M}$ and $L_{N}$ is

$$
L_{M}=k_{1} L_{N}+b_{1}
$$


where $L_{M}$ means the value obtained from the equation (14) in [1]. $k_{1}$ and $b_{1}$ represent the slope and intercept of $L_{M}=f\left(L_{N}\right)$, respectively. It should be mentioned that $L_{M}$ is the same as the value of $L$ in the Equation (3). According to the Equation (3), $L_{M}$ can be written as

$$
L_{M}=\frac{-k_{2} R_{t}}{2 b_{2}},
$$

where $k_{2}(=2 L / K)$ and $b_{2}\left(=-R_{t} / K\right)$ are the parameters of $T_{n} / n=f\left(1 / \tau_{n}\right)$. It is noted that in [1], $R_{t}$ is the ohmic resistance in circuit. Here $R_{t}$ means the resistance value before amended. If we directly replace $R_{t}$ with $R_{t}^{\prime}$ to decrease the deviation of inductance, it is hard to obtain $L_{M}$ after that $R_{t}$ is amended.
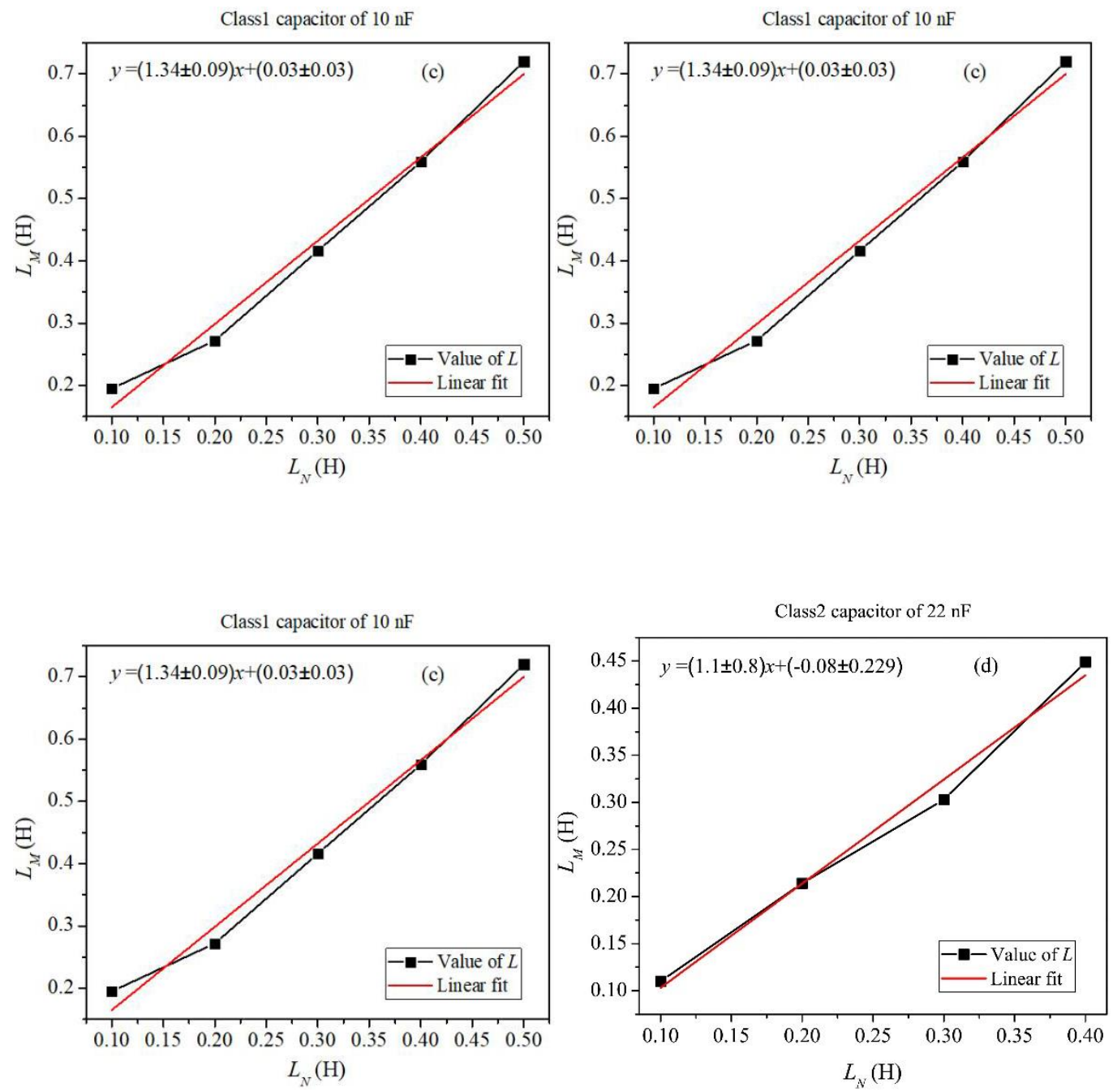

Figure 2. (color online). (a) Graph of relation $T_{n} / n=f\left(1 / \tau_{n}\right)$ of $L=20 \mathrm{mH}$. (b) Graph of relation between nominal values of $\mathrm{L}$ and measured values when Class $22 \mathrm{nF}$ connected in parallel. (c) Graph of relation between nominal values of $L$ and measured values when Class $110 \mathrm{nF}$ connected in parallel. (d) Graph of relation between nominal values of $L$ and measured values when Class $22 \mathrm{nF}$ connected in parallel. 


\section{Conclusions}

The main propose of this paper is to examine the new method of measuring ESR and the coil inductance. During the experiment, standard equipment was connected into the circuits, such as inductance coils, oscilloscope, signal generator, ceramic capacitors, particularly Class1 and Class2 capacitors. Specific phenomena appeared in the measurement. We explained the intercept in ESR and the deviation in the measuring inductance. The amended method are proposed. The ESR should be written as $E S R^{\prime}=E S R+b$. As well, there is a linear relation between measured values of $L$ and nominal values, which can be used to amend measured L.

Funding: This research was funded by the Natural Science Foundation of Guangdong Province, grant number 2016A030313433.

\section{Conflicts of Interest:}

The authors declare no conflict of interest.

\section{References:}

Ivković, S. S., Marković, M. Z., Ivković, D. Ž., \& Cvetanović, N. (2017). LCR circuit: new simple methods for measuring the equivalent series resistance of a capacitor and inductance of a coil. Eur. J. Phys., 055705, 38. 